\title{
CORRIGENDUM
}

doi:10.1038/nature24025

\section{Corrigendum: High-temperature crystallization of nanocrystals into three-dimensional superlattices}

Liheng Wu, Joshua J. Willis, Ian Salmon McKay, Benjamin T. Diroll, Jian Qin, Matteo Cargnello \& Christopher J. Tassone

Nature 548, 197-201 (2017); doi:10.1038/nature23308

In this Letter, we reported the rapid formation of superlattices at elevated temperatures and the continued growth of nanocrystals within the superlattices for metallic, ferromagnetic and semiconducting colloids, highlighting the universality of this finding by providing a theoretical framework for the observation that spans multiple colloidal systems. We regret that we inadvertently omitted to cite previous relevant work on the small-angle X-ray scattering (SAXS) investigation of gold nanocrystal superlattice formation by reduction of an $\mathrm{Au}$ (III) solution at room temperature ${ }^{1}$. This omission does not affect the results or conclusions of the Letter. We thank B. Abécassis for bringing ref. 1 to our attention. The original Letter has not been corrected.

1. Abécassis, B., Testard, F. \& Spalla, O. Gold nanoparticle superlattice crystallization probed in situ. Phys. Rev. Lett. 100, 115504 (2008). 